International Journal of Translational

Medical Research and Public Health (2019), Volume 3, Issue 2, 66-74

\begin{tabular}{|c|c|c|}
\hline & & $\begin{array}{l}\text { INTERNATIONAL JOURNAL OF TRANSLATIONAL } \\
\text { MEDICAL RESEARCH AND PUBLIC HEALTH } \\
\text { ISSN: } 2576-9499 \text { (Online) }\end{array}$ \\
\hline ITTMRPH & Available online at www.ijtmrph.org & $\begin{array}{l}\text { ISSN: 2576-9502 (Print) } \\
\text { DOI: 10.2| | 06/ijtmrph.74 }\end{array}$ \\
\hline
\end{tabular}

ORIGINAL ARTICLE | ADOLESCENT HEALTH

\title{
Adolescent and Young People's Utilization of HIV/Sexual and Reproductive Health Services: Comparing Health Facilities and Mobile Community Outreach Centers
}

\author{
Ikenna J. Nwakamma, MPH ${ }^{\bowtie I, 6}$; Carol S.Talla, BMLS²; Stephanie E. Kei, MD³; \\ Genevieve C. Okoro, BNS ${ }^{4}$; Godwin Asuquo, $\mathrm{PhD}^{1}$; Kema A. Onu, MD \\ 'Africa Center for Health Leadership, MIB Plaza Gwarimpa,Abuja, Nigeria; ${ }^{2}$ Catholic Caritas Foundation of Nigeria, Asaba, Delta State, Nigeria; ${ }^{3} \mathrm{Federal}$ \\ Medical Center, Yenegoa, Bayelsa State, Nigeria; ${ }^{4}$ Center of Excellence in Reproductive Health Innovation (CERHI), University of Benin Nigeria; ${ }^{5}$ AIDS \\ Healthcare Foundation (AHF), Abuja, Nigeria; ${ }^{6}$ NNERELA+ Nigeria, 7 I Citec Villa, $4^{\text {th }}$ Avenue, Gwarimpa, Abuja, Nigeria \\ Corresponding author email: ike.nwakamma@gmail.com
}

\section{ABSTRACT}

Background/Objectives: Demand creation for uptake of HIV and sexual reproductive health (HIV/SRH) services among adolescents and young people (AYP) in Nigeria is challenging. This study compares the reach, utilization patterns, and factors that drive the patterns of utilization of HIV/SRH services by AYP in mobile outreach service centers and health care facilities in Nigeria's capital city.

Methods: Data were obtained from service exit surveys and HIV/SRH service utilization records in selected health facilities and mobile testing outreaches from January to April 20I8. The service providers were provided a checklist to capture key information during interactions with their AYP clients. Data were captured with Microsoft Excel, imported to and analysed with Statistical Package for Social Sciences, version 16.

Results: Community-based mobile outreaches reached a significantly higher proportion of participants, with $88 \%$ of them from the community HIV testing points. Among the participants in the SRH service utilization assessment, I5\% (20) and 19\% (142) voluntarily asked for SRH-related information in the health facility and mobile outreach respectively; $40 \%$ (53), and 30\% (224) accepted offers of SRH counselling in the health facility and mobile outreach respectively. There were significant differences in the waiting time for testing and waiting time for result collection at the mobile testing posts and the health facilities.

Conclusion and Implications for Translation: AYP friendly mobile community outreach model shows more promise in terms of reach and also seems to encourage voluntary requests for HIVISRH services among AYP.The costs and waiting times favor the mobile outreach model; however, the quality in terms of personnel and environment was an issue of concern. Health facilities (including hospitals) are not providing friendly environments that encourage voluntary uptake of HIV/SRH services by AYPs. A model for AYPs should prioritize community based and friendly services with well-trained personnel in order to build the confidence of AYPs for improved SRH seeking behaviors.

Keywords: HIV • Sexual and reproductive Health • Adolescents and Young People • Preferences • Mobile outreaches services $•$ Health facility testing • Abuja Nigeria

Copyright (C) 2019 Nwakamma et al. Published by Global Health and Education Projects, Inc. This is an open-access article distributed under the terms of the Creative Commons Attribution License CC BY 4.0. 


\section{Introduction}

\section{I.I Background of the study}

According to the United Nations Children's Fund (UNICEF), worldwide there were about 590,000 young people between the ages of 15 to 24 who were newly infected with HIV in 2017 alone; among this, 250,000 were adolescents aged I5 to 19 years.' AIDS-related deaths among adolescents tripled in the 15 years from 2000 to 2015 while decreasing among all other age groups, which can be largely attributed to a generation of children who were vertically infected during infancy. ${ }^{2}$

Nigeria has a population of over 173 million people, with those aged 10-14 contributing $12.3 \%$ of the population and 15-19, and 20-24 year olds constituting $8.8 \%$, and $7.2 \%$ respectively. ${ }^{3}$ Although there is a paucity of data on national prevalence of sexually transmitted infections (STI) in Nigeria, there are some state-level studies that provide useful information on the patterns of STI among young people. A study in Nigeria's capital city on young people in tertiary institutions reported the prevalence of STls to be $70.60 \% .{ }^{4}$ Another study in Eastern Nigeria reported the prevalence of STIs to be $13.9 \%$ among those aged II-25 years and $17.16 \%$ among tertiary school students aged 16-40 years. ${ }^{5}$ The 2019 Nigeria's AIDS Indicator Impact Survey (NAIIS) Report indicated that out of the estimated I.9 million people living with HIV in Nigeria, $0.2 \%$ are aged between $0-14$ years and I.5\% are aged 15 64 years. $^{6}$

Underutilization of HIV and other sexual and reproductive health $(\mathrm{SRH})$ services results in late diagnoses, particularly for young people. Demand creation for uptake of HIV and SRH services among adolescents and young people (AYP) is still a considerable challenge in Nigeria. In dealing with this problem, Nigeria is utilizing multiple approaches, including client-initiated and provider-initiated counselling and testing, both at health facilities and in communities.

Sexual and reproductive health and HIV testing services are predominantly undertaken in health facilities, but available data suggest that clients also have the option to test in the community, including home-based and mobile services. ${ }^{7}$ It is believed that community-based testing outreaches have the potential for reaching HIV-infected individuals earlier in their disease progression, ${ }^{8}$ improving health outcomes, and reducing costs of care provision. ${ }^{9}$ Mobile testing services, when conducted properly, have proven to be very efficient and cost-effective in reaching out to more people when compared to a facility-based testing approach which is offered to clients in health facilities. ${ }^{10}$

The purpose of this study was to comparatively capture data from mobile HIV/SRH service centers and focused-approach health facilities to understand the reach, utilization patterns, and factors that drive the patterns of utilization of services to AYP.

\section{I.2 Objectives of the study}

The objectives of this study were to: to compare HIV/ SRH services provided to AYP by community-based mobile outreaches vs. health facilities regarding: (I) the reach of services; (2) prevalence of voluntary requests for services; and (3) the quality and cost of accessing services. Our overall goal was to provide data for evidence-informed public health programming and policy on AYP healthcare delivery in Abuja, Nigeria.

\section{Methods}

This was a multicenter study conducted from January to April 2018 in two Local Government Areas - (I) Abuja Municipal Area Council (AMAC) and (2) Bwari Area Council within the Federal Capital Territory, (FCT), Abuja, Nigeria. The study included AYPs aged 13-24 who accessed HIV/SRH services in specific health facilities and mobile community HIV outreach sites. The specific study sites included five selected health facilities: 3 in AMAC and 2 in Bwari; and ten mobile HIV testing service outreach sites: 2 each in the same location as each of the identified health facilities.

\section{Data collection}

Data came from two sources: (I) a service exit survey questionnaire, and (2) services utilization checklist. Both were used in the health facilities and mobile outreach service points. The service providers were provided a simple checklist to capture key 
information on characteristics of all the AYP clients who used their HIV testing services within the study period and whether they requested/accepted SRH services or not. The study did not look at the screening results of the AYPs.

For the service exit survey, data were collected by waiting for the clients as they were leaving the service points. The questionnaire sought information on AYP clients' opinions on quality of the services they received and recommended preferences and cost of reaching the service points from their homes. The same tools were used for the clients at the health facilities and mobile community outreach sites.

\section{I Study variables}

The variables measured in this study include the following:

- Health facility based service: This was defined as a hospital or clinic where health services were provided;

- Mobile community outreach service: This was defined as a service provided in the community outside a health facility. In this case, it was HIV testing outreach points sited in the communities;

- Voluntary demand for HIVISRH service: This represents client-initiated discusions or requests for any form of HIV/SRH service. It could be in the form of request for screening, request for information, or request for products like condoms;

- Acceptance of SRH service:This represents client's willingness to accept screening or counseling on products like condoms and other contraceptives; and

- Quality of HIVISRH service: This is defined as the captured information of the AYP's opinion on the services provided in the different models in terms of capacity/disposition of the personnel, waiting times, confidentiality, and conduciveness of the environment.

\subsection{Statistical analysis}

Data collected from all selected facilities and mobile testing points were collated into a Microsoft Excel spreadsheet, and the aggregate number of AYP clients seen during the study period was generated and information on their service utilization patterns documented. The data was stratified by location, and by models (mobile outreach or facility-based based). The dataset was imported into Statistical Package for Social Sciences, version 16 for analysis."

Descriptive statistics were carried out producing simple tables on the patterns of HIV/SRH service uptake, disaggregated according to age groups (13-I7, I8-25), sex and locations. Chi square test was used to compare means and logistics regression was conducted to determine associations.

\subsection{Ethical clearance}

Formal ethical approval was not obtained from any ethical board as this was not designed from onset as a research project but a project implementation. However, comprehensive information was provided to adolescents and their parents or guardians in English language or their own local languages, such as Hausa, Ibo, Yoruba, TIV, Gwagi, Idoma, Igede, Ibira etc. For AYPs under 18 years, the parents/ guardians signed consent forms or orally assented for the adolescents' participation, while adolescents aged 18 years and above signed their own consent forms. The process was conducted in accordance with provisions of the standard for privacy and confidentiality guidelines for research involving young people, as recommended by British National Children's Bureau guidelines for research involving children/young people. ${ }^{12}$

\section{Results}

\section{I Reach of community-based mobile community outreaches and health facility-based services in providing HIVISRH services to adolescents and young people}

A total of 1,989 AYP were involved in this study (male, 48.7\%), out of which 120 participated in the service exit survey that assessed the opinions of the AYP on quality of services in both health facility and mobile community outreach points and their preferences. Participants aged 13-17 totaled 760 (38\%), while those aged 18-25 totaled I,229 (62\%). All participants received HIV testing services; and $\mathbf{8 7 2}$ were involved in the SRH service uptake assessment. As presented in table I, I,757 (88\%) of study participants were reached through mobile service outreaches; I,048 (53\%) participants were 
residents of the peri-urban areas while 94I (47\%) constituted rural dwellers.

\subsection{Voluntary request for HIVISRH services among adolescents and young people in health facilities and mobile community outreaches}

Table 2 presents the patterns of SRH service uptake in the health facilities and mobile outreaches. Out of the 872 AYP that participated in the assessment on $\mathrm{SRH}, \mathrm{I} 32$ (I5\%) were recorded in the health facilities, while 740 (85\%) were reached in the mobile service outreaches.Among those who reached on the health facilities, $55 \%$ (73) were females and $45 \%$ (59) were males. While those who arrived in mobile outreach constituted 54\% (399) females and 46\% (34I) males.

Voluntary uptake of SRH services was low in both health facilities and mobile service outreaches. According to the different service models, 15\% (20) and $19 \%$ (142) respectively voluntarily asked for SRH related information in the health facility and mobile outreach, while $40 \%$ (53) and 30\% (224) respectively accepted offers of SRH counselling in the health facility and mobile outreach.

On voluntary request for condoms, none was recorded in the health facilities, but in the mobile service outreaches, $3 \%$ of the females and $17 \%$ of the males asked for a condom voluntarily. When offered without request, no female accepted the offer of condoms in the health facility, while $25 \%$ of the males in the health facility accepted offers of condoms. In contrast, $9 \%$ and $53 \%$ of females and males, respectively, in the mobile outreaches accepted offer of condoms. A similar pattern was observed on the ability to access condoms when needed. While no female reached in the health facility acknowledged to have ever bought a condom or willing to request condoms from a retailer, $20 \%$ of the males in health facility confirmed that they have bought a condom; $3 \%$ of the females and $14 \%$ of the males in mobile outreaches accepted to have bought a condom from a retailer.

On the willingness to request condoms from a retailer when needed, $39 \%$ of the males in the health facilities, $7 \%$ and $18 \%$ of the females and males respectively in mobile outreaches responded in the affirmative. A different pattern was observed on possible uptake of syphilis screening. While more of the females, (100\% in health facilities and $72 \%$ in mobile outreaches) expressed willingness to accept syphilis test when offered free, more of the males expressed ability to pay for the test when recommended.

\subsection{Quality and cost of accessing HIVISRH services in health facilities and mobile community outreaches}

From the service exit surveys, we captured the experiences and opinions of the AYP who were reached

Table I. Patterns of HIV Counselling and Testing (HCT) Uptake in the health facilities and mobile service posts by gender and age groups

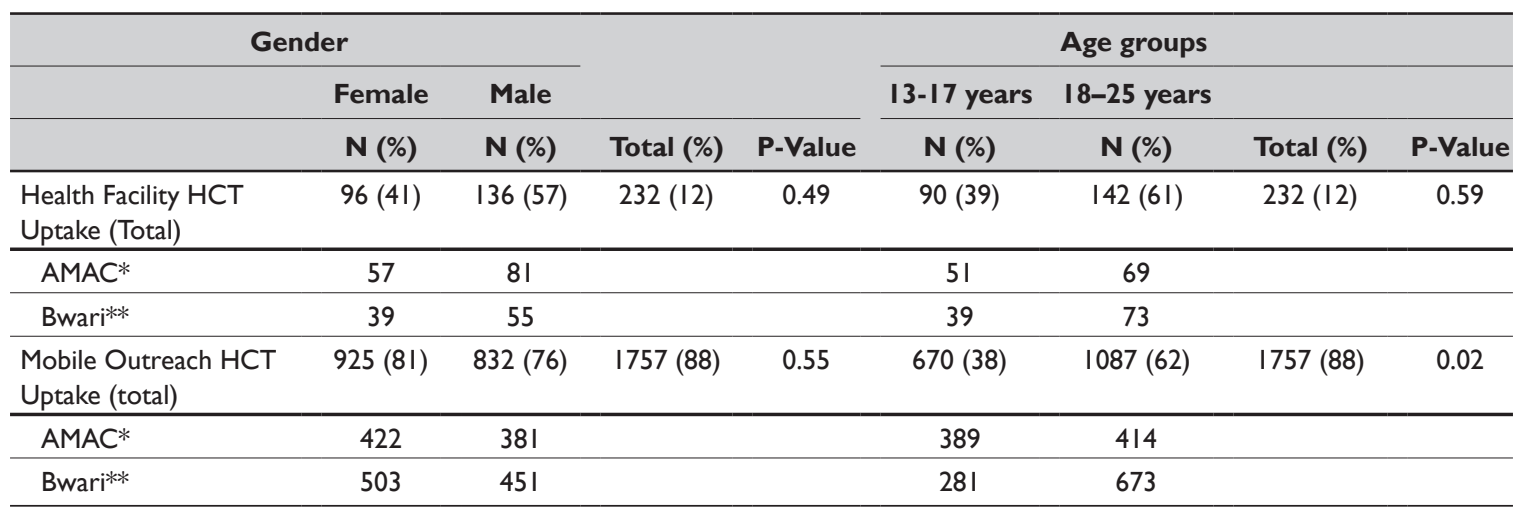

*Represents the urban residents

**Represents the peri-urban resident 
at the different service points. The exit surveys focused on appropriateness of the location of the health facility and mobile outreach points. It also focused on the issue of confidentiality of the sessions and spaces for HIV counselling and testing (HCT). Other areas examined were the attitude of the facility staff, cost of accessing HCT, and waiting times for testing and collection of test results. Data from service exit survey (Table 3) captured the opinions and experiences of the AYP according to the models of HCT they accessed (health facility versus mobile outreach testing). User opinions were captured on the scale ranging from "unsatisfactory," "good" and "very good."

Table 4 presents the responses on waiting times for test and results at the health facility and mobile testing outreaches. There were significant differences in the waiting times for testing and waiting times for result collection at the mobile testing posts and the health facilities $(p=0.04$ and $p=0.02$, respectively). The responses of the AYP at the health facilities and mobile outreach testing points showed that $79 \%$ of those who went to test at mobile outreach points tested and received their results within 30 minutes, while only $30 \%$ of those who went to health facilities were able to test within the same time frame. On the cost of reaching the service points, average response among those reached in the mobile outreaches was I 20 Naira (equivalent: $\$ 0.34$ US), while those reached in health facilities was more than double specifically, 254 Naira (approximately $\$ 0.7$ I US).

\section{Discussion}

\section{I Discussion}

The need for effective and efficient SRH/HIV programming for AYP has become more pertinent, particularly as many infants with perinatal exposure to HIV have grown to adolescence and early adulthood. These young people will interact with and develop sexual relationships with their peers. Another source of worry is the increased migration of young people into urban areas, where they are faced with so many realities of the urban setting like adolescent sex work, different moral values, child labor, economic hardship, sexual abuse, coercive sex

Table 2. Pattern of sexual reproductive health (other than HIV) service Uptake in Health Facilities and Mobile Service Points According to Gender

\begin{tabular}{|c|c|c|c|c|}
\hline & \multicolumn{2}{|c|}{ Health Facility } & \multicolumn{2}{|c|}{ Mobile Testing points } \\
\hline & Female & Male & Female & Male \\
\hline & $\mathbf{N}(\%)$ & $\mathbf{N}(\%)$ & $\mathbf{N}(\%)$ & $\mathbf{N}(\%)$ \\
\hline Total & $73(55)$ & $59(45)$ & $399(54)$ & $34 \mid(46)$ \\
\hline $\begin{array}{l}\text { Voluntary request for SRH } \\
\text { information* }\end{array}$ & $7(10)$ & $13(22)$ & $53(13)$ & $89(26)$ \\
\hline Accepted SRH counselling ** & $24(33)$ & $29(49)$ & $73(18)$ & I5I (44) \\
\hline Voluntary request for condoms & 0 & 0 & $12(3)$ & $58(17)$ \\
\hline Accepted offer of condom & 0 & $15(25)$ & $36(9)$ & $182(53)$ \\
\hline Knows a place to get/buy condom & $28(38)$ & $41(69)$ & $240(60)$ & $311(91)$ \\
\hline Ever bought a condom & 0 & $12(20)$ & II (3) & $47(14)$ \\
\hline $\begin{array}{l}\text { Willingness to request for condom } \\
\text { from a retailer when needed }\end{array}$ & 0 & $23(39)$ & $27(7)$ & $62(18)$ \\
\hline $\begin{array}{l}\text { Willing to accept syphilis screening if } \\
\text { offered free }\end{array}$ & $73(100)$ & $49(83)$ & $288(72)$ & $211(62)$ \\
\hline $\begin{array}{l}\text { Can pay for syphilis test if } \\
\text { recommended }\end{array}$ & $20(27.4)$ & $31(52.5)$ & $97(24.3)$ & $103(30.2)$ \\
\hline
\end{tabular}


Table 3. Adolescents and young peoples' opinions on quality of service in the different service models

\begin{tabular}{|c|c|c|c|c|c|c|}
\hline & \multicolumn{3}{|c|}{ Health Facility } & \multicolumn{3}{|c|}{ Mobile Testing points } \\
\hline & Not satisfactory & Good & Very good & Not satisfactory & Good & Very good \\
\hline Location & $4(10 \%)$ & $28(70 \%)$ & $8(20 \%)$ & $21(29 \%)$ & $38(53 \%)$ & $13(18 \%)$ \\
\hline Confidentiality & $13(32 \%)$ & $21(52.5 \%)$ & $6(15 \%)$ & $10(14 \%)$ & $4 \mathrm{I}(57 \%)$ & $21(29 \%)$ \\
\hline Staff attitude & $8(20 \%)$ & $15(37 \%)$ & $17(43 \%)$ & $5(7 \%)$ & $52(72 \%)$ & $15(21 \%)$ \\
\hline
\end{tabular}

Table 4. Estimated waiting times and average cost of reaching a testing center and for testing/result collection.

\begin{tabular}{|c|c|c|c|c|}
\hline & Mobile HCT Center & $\begin{array}{l}\text { Health } \\
\text { Facility }\end{array}$ & $\mathbf{X}^{2}$ & P-value \\
\hline \multicolumn{5}{|l|}{$\begin{array}{l}\text { Estimated waiting time before } \\
\text { testing }\end{array}$} \\
\hline$\leq 30$ minutes & $57(79 \%)$ & $12(30 \%)$ & & \\
\hline $30 \mathrm{~min}-1$ hour & $15(21 \%)$ & $18(45 \%)$ & 6.22 & 0.04 \\
\hline$>$ Ihour & 0 & $10(25 \%)$ & & \\
\hline \multicolumn{5}{|l|}{ Estimated waiting time for result } \\
\hline$\leq 30$ minutes & $57(79 \%)$ & $5(12.5 \%)$ & & \\
\hline $30 \mathrm{~min}-1$ hour & $15(21 \%)$ & $14(35 \%)$ & 7.52 & 0.02 \\
\hline$>$ Ihour & 0 & $21(52 \%)$ & & \\
\hline $\begin{array}{l}\text { Average cost of transport to and from Services point } \\
\text { (in Naira) }\end{array}$ & 120 & 254 & & \\
\hline
\end{tabular}

with older persons, and loneliness that may drive psychological vulnerability.

The opinions, perceptions and preferences of AYP on HIV counselling and testing and SRH services were captured to address the issues around knowledge, access to condoms, and also their preferences between facilities-based and mobile outreach services. One major influence of risky sexual behaviour is lack of HIVIAIDS prevention knowledge among adolescents. ${ }^{13}$ A prior study on the effects of curriculum-based sex and HIV education programs on sexual behaviour found that within the United States, the majority of education programs were effective in delaying onset of sexual activity and increasing condom use among young people, ${ }^{14}$ suggesting that through reproductive health education programs, which enhance one's knowledge, young people can be encouraged to engage in safer sexual behaviours.

It is important to point out that knowledge of where to get condoms was relatively higher among the males than females and was expressed more in the mobile services interactions in both sexes. This pattern of gender imbalance in access to information on condoms among adolescents and young women has been observed in a prior study. ${ }^{15}$ Condoms have been acknowledged to provide dual protection against unwanted pregnancy and transmission of STls; use of condom is recognized as the most potent way to prevent the transmission of HIV sexually. Though males commonly use condoms, they are usually the ones in control of HIV transmission. The knowledge among females will help in negotiating safer sex and proper use of condoms among the sexually active females. It should be noted also that the observed low knowledge was among those that were reached through health facilities, pointing out that the female AYP may not be finding the health facilities as the avenues for meeting their SRH needs.

On preferences between the service models, more AYPs were reached in the mobile community 
outreach services. However, there was higher expression of satisfaction with the location and confidentiality among those that used health facilities compared to those who used community mobile outreaches. On the other hand, AYPs who were reached at the mobile outreaches expressed more appreciation for staff attitude. This was also evident on the levels of voluntary request for condoms and $\mathrm{SRH}$ information in both models. For instance, while there was no voluntary request for condoms in the health facilities, about $20 \%$ of those who were reached in the mobile service points requested condoms. Studies have reported that healthcare workers' negative behaviors discourage young people, particularly the females, from accessing SRH services in hospitals. ${ }^{16,17}$ This further supports the call for a friendlier healthcare delivery system for AYPs in the healthcare facilities.

Evidence suggests that one of the strongest predictors of satisfaction with services included accessibility, convenience and availability. ${ }^{18,} 19$ The comparative effectiveness of accessing health facility based testing and mobile outreach testing was assessed using service exit data from the points of service. It was observed that a good proportion of participants who accessed mobile HCT reported shorter waiting times before seeing a counsellor and also receiving results. Waiting time is a major concern for clients who only want to access HCT, and prolonged waiting time is associated with higher levels of dissatisfaction with services. ${ }^{20}$ Comparatively, participants incurred lower cost in transportation and shorter travel time in the mobile HCT due to the fact that the services were brought close to their places of residence or work. Cost, in terms of time and money, associated with accessing services as well as longer travelling distances and waiting time have been implicated as impediments to use of services. ${ }^{21} \mathrm{~A}$ high point for mobile testing is the ability to reduce travel time and costs due to the high proportion of "opportunistic visits" that see it as a quick opportunity to know their HIV status.

In this study, we show that mobile HCT approach was able to reach a significantly higher proportion of participants, including those that had no initial plan of accessing the services but were able to take advantage of the opportunity presented to them. This observation underscores the importance of taking the services to the people to bridge the gap in healthcare access, particularly for underserved communities.

\subsection{Limitations}

It is important to note that this study did not look at positivity yield in the different testing models as the major objective was to identify which model best appeals to the AYP. Also, SRH/HIV services seeking behavior for health facility and mobile outreach services may not be rightly compared in this study as most of the AYP reached in the health facilities were through provider-initiated HIV testing. Majority of them reached at the health facility came for other healthcare needs; therefore, their responses may be biased due to their desire to comply with their treatment processes and thus overestimate their actual desires to voluntarily uptake the services. Future studies should include qualitative assessment to have some in-depth naratives on the opinions of the AYP and clearer understanding of the reasons behind their preferences.

\section{Conclusion and Implications for Translation}

Health programming for AYP should be based on evidence, and in this study the young people expressed their preferences. It is therefore important to put the issues in focus while intervening on $\mathrm{SRH} / \mathrm{HIV}$ among the young people. Health facilities remain important in the management of HIVI STI as they provide a pool of qualified personnel and ensure adequate capture of HIV/STI related data. Also, because the health facilities are in fixed locations, AYP who tested positive to HIV/STI and have passed the stage of denial can still walk back to the health worker for psychosocial support. This is unlikely for a mobile testing outreach. However, taking the HIV/STI response into the communities is very vital in the fight against the determinants of HIV/STI infection like stigma, access to information and early diagnosis for prevention of mother to child transmission. Mobile HCT services provide the opportunity to reach high risk spots and highly populated locations like workplaces, bus stops and 
within communities. However, mobile HCT services should run in conjunction with health facility services for effective referral, follow-up and data reporting.

\section{Compliance with Ethical Standards}

Conflicts of Interest: All the authors hereby declare that there was no conflict of interest that may have affected the results obtained in the study. Financial Disclosure: The authors hereby declare that there was no financial compensation received for this study. Funding/Support: The funding of the study was through personal contributions of the authors. Ethics Approval: Written/verbal consents were obtained from all participants; written/verbal consent was obtained from parents/care givers of participants below the age of 18 . Acknowledgments: None. Disclaimer: None.

\section{References}

I. United Nations Children's Fund (UNICEF). Turning the tide against AIDS will require more concentrated focus on adolescents and young people. Adolescent HIV Prevention. https://data.unicef.org/topic/hivaids/ adolescents-young-people. Published July 2019. Accessed July 28, 2019.

2. United Nations Children's Fund. Annual Results Report 2015 HIV and AIDS. UNICEF;20I6.

3. Porth, T, Suzuki C, Gillespie A, Kasedde S, Idele P. Disparities and trends in AIDS mortality among adolescents living with HIV in low- and middleincome countries. Paper presented at: 20th

\section{Key Messages}

Provision of HIV and other sexual and reproductive health services for adolescents and young people must not be limited to the hospitals; youth friendly centers may be preferable to encourage increased uptake of these services by the young people.

Although, girls/young women bear more burden of HIV and other sexually transmitted infections, they are less economicaly and socially empowered to access the required services. Adolescents and young people need safe spaces for proper expression of their sexual and reproductive health needs.
International AIDS Conference; July 20-25, 20I4; Melbourne, Australia.

4. National Population Commission, ICF International. Nigeria Demographic and Health Survey 2013. NPC and ICF International; 20I4.

5. Obiajuru OC, Jude NO. The prevalence of sexually transmitted infections among post-primary and tertiary school students in Imo State, Nigeria. Niger J Health Bio Sci. 2007;6(2):90-5 doi: 10.43|4/njhbs. v6i2. II65I

6. Nigeria HIV/AIDS Indicator and Impact Survey (NAIIS). National Summary Sheet: Preliminary Findings. Federal Ministry of Health Nigeria; 2019.

7. Staveteig S, Wang S, Head S, Bradley SEK, Nybro E. Demographic Patterns of HIV Testing Uptake in SubSaharan Africa. ICF International; 2013.

8. Morin SF, Khumao-Sakutukwa G, Charlebois ED, et al.J Acquir Immune Defic Syndr. Removing barriers to knowing HIV status: same-day mobile HIV testing in Zimbabwe. 2006;4I(2):218-224. doi: 10.1097/0I. qai.0000179455.01068.ab

9. MacPherson P, Corbett EL, Makombe SD, et al. Determinants and consequences of failure of linkage to antiretroviral therapy at primary care level in Blantyre, Malawi: a prospective cohort study. PLoS One. 2012;7(9):e44794. doi: 10.1371/journal. pone.0044794

10. Wachira J, Kimaiyo S, Ndege S, Mamlin J, Braitstein P. What is the impact of home-based HIV counseling and testing on the clinical status of newly enrolled adults in a large HIV care program in Western Kenya? Clin Infect Dis. 2012;54(2):275-28I. doi: 10.1093/cid/cir789

II. SPSS Inc. Released 2007. SPSS for Windows, Version 16.0. Chicago, SPSS Inc.

12. Shaw C, Brady L, Davey C. Guidelines for Research with Children and Young People. National Children's Bureau Research Center; 201 I.

13. Bazargan M, Kelly EM, Stein JA, Husaini BA, Bazargan SH. Correlates of HIV risk-taking behaviors among African-American college students: the effect of HIV knowledge, motivation, and behavioral skills. J Natl Med Assoc. 2000;92(8):391-404.

14. Andrade SS, Zaccara AA, Leite KN, et al. Knowledge, attitude and practice of condom use by women of an impoverished urban area. Rev Esc Enf USP. 20I5;49(3):364-37I. doi: I0.1590/S0080623420150000300002 
15. Glasier A, Gulmezoglu AM, Schmid GP, Moreno CG, Van Look PF. Sexual and reproductive health: a matter of life and death. Lancet. 2006;368(9 547): I 595-I 607. doi: I0.1016/S0I40-6736(06)69478-6

16. Mbadu Muanda F, Gahungu NP,Wood F, Bertrand JT. Attitudes toward sexual and reproductive health among adolescents and young people in urban and rural DR Congo. Reprod Health. 2018;15(I):74. doi: |0.1 |86/s | 2978-0| 8-05 I7-4

17. Anastasi M, Sawyer RG, Pinciaro PI. A descriptive analysis of students seeking HIV antibody testing at a university health service. J Am Coll Health. 1999;48(I): I3-19. doi: I0.1080/07448489909595667

18. Nabbuye-Sekandi J, Makumbi FE, Kasangaki A, et al. Patient satisfaction with services in outpatient clinics at Mulago hospital, Uganda. Int J Qual Health Care. 20 I I;23(5):5 I6-523. doi: I0.1093/intqhc/mzr040
19. Wouters E, Heunis C, van Rensburg D, Meulemans $\mathrm{H}$. Patient satisfaction with antiretroviral services at primary health-care facilities in the Free State, South Africa--a two-year study using four waves of crosssectional data. BMC Health Serv Res. 2008;8:210. doi: 10.1186/1472-6963-8-210

20. Mubyazi GM, Bloch P, Magnussen P, et al. Bygbjerg IC Women's experiences and views about costs of seeking malaria chemoprevention and other antenatal services: a qualitative study from two districts in rural Tanzania. Malar J. 2010,9:54. doi: 10.1 186/1475-2875-9-54

21. Leisegang R, Cleary S, Hislop M, et al. Early and late direct costs in a Southern African antiretroviral treatment programme: a retrospective cohort analysis. PLoS Med. 2009;6(I2):el000189. doi: 10.137|/journal.pmed. 1000189 